\title{
Survey on cryptographic block cipher methods to solve the security issues
}

\author{
DAYANANDA LAL $\mathrm{N}^{\# 1}$, Dr. SENTHIL KUMAR.K ${ }^{* 2}$ \\ \#1 Research Scholar, Department of Electronics and Communication Engineering, \\ Dr. MGR Educational and Research Institute University, Chennai, India \\ Email id: dayanandlal@gmail.com \\ ${ }^{* 2}$ Professor, Department of Electronics and Communication Engineering, \\ Dr. MGR Educational and Research Institute University, Chennai, India \\ Email id: ksenthilkumar@drmgrdu.ac.in
}

\begin{abstract}
Paper concentrates on surveying a key region of information systems administration hypothesis - The Open Systems Interconnect (OSI) 7 Layer Model. This survey exhibits the utilization of the prototype ideas into the setting of data safety. This survey shows the point of view that normal data security issues outline to the sensible builds displayed in the OSI 7 Layer Network Model. A couple layers have more impact than others while securing information. Jointly, they can be utilized to fabricate a complete arrangement. Using the OSI Model's 7 layers, this survey paper will exhibit an intelligent, complete and achievable way to deal with securing an association's data assets. In this paper, creator gives a short outline of Symmetric key block cipher for various calculations exhibited in this field as per characterized it in cryptography where we ordered into classes. To start with, Mode of operation which is routes connected block cipher to encode bigger plaintext. Second, iterated item cipher which additionally ordered it into Feistel Network, substitution-stage organizes and Unbalanced Feistel Network.
\end{abstract}

Keyword -OSI (Open system Interconnection), SSL (Secure Sockets Layer), DES (Data Encryption Standards), AES (Advanced Encryption Standards), FEAL (Fast data encipherment algorithm), CLEFIA

\section{INTRODUCTION}

\subsection{OSI Network Layer Introduction}

Networking is a prime sympathy toward data security. [1] The pervasive way of system availability may give us a chance to get to the world from our PC; however, it additionally gives that same world pick up a chance to access back to us in ways we may not pine for. Despite how well we secure our own particular hosts, we are still frail if the parts of the establishment between our far away objectives and ourselves capitulate to ponder mishandle or unwitting episode. Information security and data systems administration are indistinguishably associated subjects. Today's framework configuration must pick the alternative to be security-discerning, and the security construct must pick the choice to grasp the framework he is endowed to secure.

A ton of formalized audit has been focused on the science and arrangement of arranging and caring for frameworks. One formal structure that framework engineers discuss and apply as frequently as conceivable is the OSI Seven Layer Model for Networking, made by the ISO to characterize an institutionalized strategy for planning systems and the capacities that bolster them. This prototype portrays 7 layers of cooperation for a data framework conveying over a system, showing a heap of layers speaking to significant capacity ranges that are for the most part required or helpful for information correspondence between hubs in a circulated situation. Beginning from an abnormal state application viewpoint, information is sent down the stack layer by layer, every layer including data around the initially displayed information until that unique information in addition to its layers of included substance are spoken to at the bottommost layer as a optical fibre, for example, blasts of hued light or voltage over a wire all together for that information to optically head out from one indicate the other in this present reality.

1.2 OSI Layer

In [1] Damon read tells about the OSI 7-layer prototype to data security. Information systems administration is a basic territory of centre in the investigation of data security. Creator concentrates on evaluating a key territory of information systems administration hypothesis - OSI Seven Layer Network Model. This survey paper exhibits the use of the model's ideas into the setting of data security. This paper general shows the point of view that regular data_security issues delineate to the coherent builds exhibited in the OSI 7 Layer Network Model, and looks to show the Seven Layer Model's handiness in assessing data_security issues and arrangements. The OSI_Model is displayed by method for the two-conventional denotation and pragmatic terms that influence data security on a layer_by_layer premise. 
For every coating, cases of basic data surety dangers and rules are assessed by how they suitable into the OSI 7 Layer Model's order, with notations on exemptions and extraordinary occurrences. Formarly the 7 layers have been secured as a reason to talk, it is exhibited that the 7 Layer Model's plan for cooperation joining the skin offers knowledge to a portion of the issues confronted by engaged, "single_layer" security arrangements. To answer these issues, a multiple_layer "resistance top to bottom" resemble is analysed via illustration, catch from the perspective of system imitation layers as opposed to individual arrangements along with sensible or substantial equipment layers. The creator closes with some advanced expansions to the prototype that total the prototype's supplication to data security issues.

In [2], Kari A. Pace clarifies the layered security show. Data is the heart of any manufacturing company. It gives nourishment to authoritative peoples: enabling also with reinforcing its clients via gatherings and people. It can be utilized for or against us, normally favouring us with the wellbeing and trustworthiness of our data. In the event that the way of our data is to be appropriated for openness then so should our Endeavor's to make safe. In the course of recent decades, the conveyed registering company has used the ISO OSI_Model for better institutionalization of equipment and programming segments. A few layers have more effect than others while securing data. Together, they can be utilized to assemble a far-reaching arrangement. Using the OSI Model's seven layers, creator will exhibit an intelligent, far reaching and achievable way to deal with securing an association's data assets.

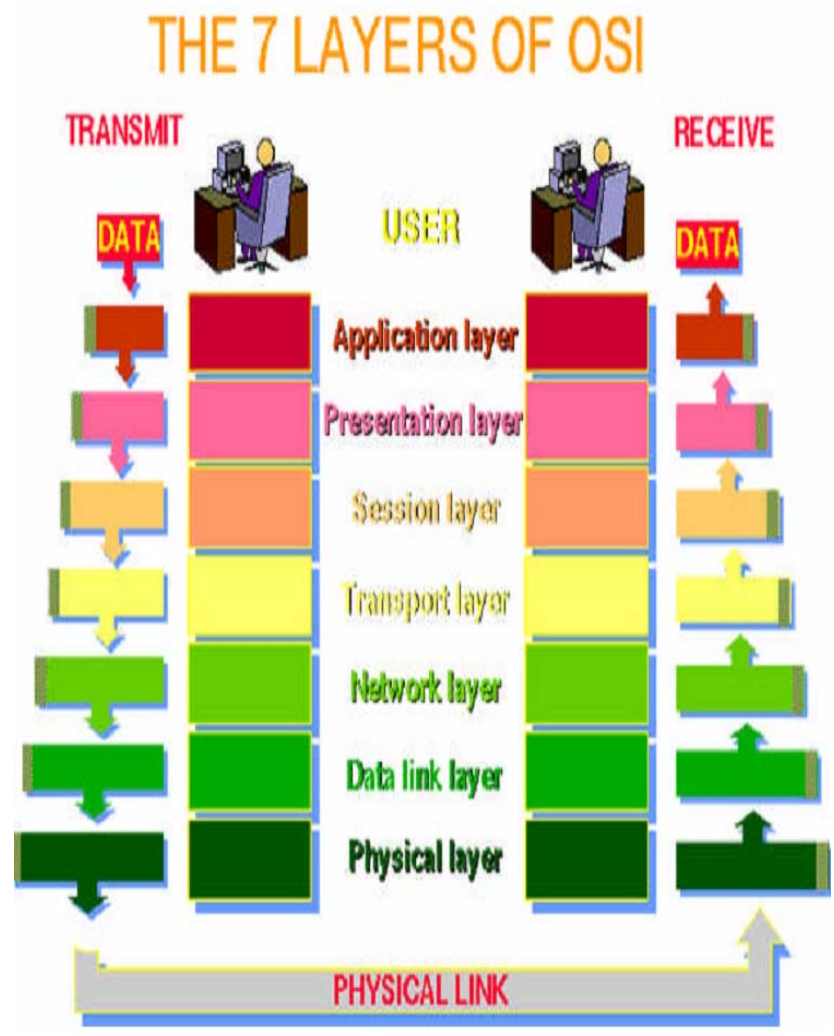

Fig 1: Seven Layers of OSI Model

In this paper [2], the OSI Layers Shown in above fig 1

Physical Layer

The sensible initial phase in shield, which given data for guaranteeing the physical assets which never traded off. The physical layer of the OSI_Model reminds us to not disregard the self-evident. Frequently, engineers neglect to perceive the significance of the basic estimates, as appropriately bolting stockpiling units, server cupboards, hardware rooms and office spaces. Accessing assets is the initial phase in bargaining them.

Physical latch, together on hardware and offices lodging the gear are basic to hold gatecrashers out. Keeping in mind the goal to utilize the data should have admittance to it. Safety links on tablets and framework indexes with command catch latches are cases of acquiring gear with physical security capacities. 


\section{Data Link Layer}

The Data Link layer of the OSI_Model is darker than its antecedent. Layers duty shows to locate outlines onto the system channel and protect that conveyance is without blunder. This is the place the Medium Access Control (equipment) address of specialized gadgets is used and bitmap for mistake in conveyance are connected. A gadget racing in wanton way together a parcel channel perhaps useful otherwise hurtful instruments at OSI_Layer. Permitting stream investigation, issue assurance and code troubleshooting can be useful. Nonetheless, in the wrong hands the capacity to duplicate datagrams represents a risk.

Network Layer

Some switches work at Layer 3 of the OSI Model, despite the fact that cynicism of its prosperity still proliferates. As a general rule, we will discover switches and firewalls working this layer. The best way at this layer is resolved from starting point to goal have on a system.

\section{Transport Layer}

Computing a framework on the web need to know the general population IP delivers allocated to this. To focus on a particular request on a framework, a gatecrasher is to know the IP deliver to find the framework and the port no relegated to the request, by and large alluded to as an attachment. A PC framework has 65535 ports. These ports can be further separated into three classes: surely understood, enlisted and dynamic. This is the place Layer 4 security is connected. Numerous applications use surely understood TCP and UDP. A FTP server will, as a matter of course, use TCP port 21 . On the off chance that the record server giving the FTP administration is not implied for open space, the best part is to change the port no and unveil the new port no to approved clients as it were. Along these lines, we can befuddle and slow down potential interlopers by utilizing private ports as a part of place of surely understood ports.

\section{Session Layer}

Layer 5 of the OSI demonstrates manages session taking care of between frameworks. Its occupation is to encourage correspondence with an accepting gadget by building up, looking after, synchronizing, controlling and ending associations. Amid this procedure of correspondence, confirmation of substances can happen. Likewise alluded to as Transport-Layer-Security, Secure-Socket-Layer(SSL) is an innovation intended to affirm the character of presenter and retainers. In spite of the fact that called Transport-Layer-Security, this capacity rests simply over the vehicle layer and is really session-layer based. The indefinite quality with the OSI or more is reason for their aggregate citation 'upper-layers'. SSL is frequently the convention utilized for secure charge id exchanges on the web. Utilizing retainer validation, a retainer's personality can be checked by a CertificateAuthority(CA) utilizing Public-Key-cryptography. This can be connected utilizing customer part verification.

Presentation Layer

Encipher administrations are connected with the Outer Layers of the OSI demonstrate, particularly the Presentation Layer. Information is taken at this point, what frame it takes? Encipher systems permit us to scramble the parcel substance, need an extraordinary point to uncover them. The further modern the encipher calculations; it is tough to access the data. Clearly, serious preparing capacity might influence framework execution. Legitimate arranging is important to ascertain security needs and adjust them with asset impediments.

\section{Application Layer}

At long last, the last layer of the OSI show alludes to the supplications that bolster the last client capacities. No mistaken for client programming, supplications at this layer incorporate FTP, SMTP and other administrations. At this point, where administrations bolster client supplications that verification happens. The Frequent wellknown type of validation is user id and secret key. In this situation, each client has an extraordinary card and classified secret word. The blend of both gives the client gets to. In this way, it is basic to have a compelling record strategy.

\subsection{Cryptography}

Shelley Kandola [3] review an assortment of cryptographic calculations falling into four fundamental classifications: DES(block-cipher), RC4(stream-cipher), SHA-1(hashing), and RSA (open key cryptography). The Author gives a Python execution to each of these calculations. Cryptographic calculations are a method for cautiously passing on mystery data between one gathering and another. When all is said in done, a plaintext message is scrambled utilizing a cryptographic calculation. Through encryption, the first message gets to be ciphertext and its unique substance is totally hidden. The ciphertext can then be sent securely to the beneficiary. At the point when the beneficiary is prepared to uncover the message, he or she can do as such by applying an unscrambling calculation, which will uncover the first plaintext. Just the beneficiary can apply the unscrambling calculation in light of the fact that, in a perfect world, just the beneficiary knows the keys fundamental for decoding the ciphertext. Keys are utilized to customize and secure a cryptographic calculation to just the sender and beneficiary. 


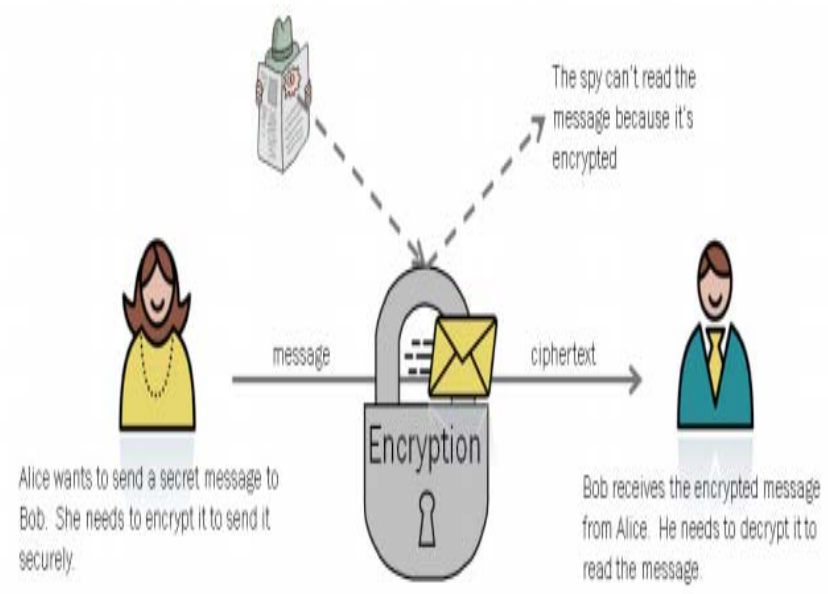

Fig 2: Concept of Cryptography

Secure encryption requires two properties: confusion and diffusion. Confusion alludes to entangling the connection via ciphertext and the key however much as could be expected though diffusion alludes to muddling the relationship amongst plaintext and ciphertext however much as could reasonably be expected.

One approach to accomplish confusion is through substitution: the supplanting of characters with different characters. The Caesar cipher, for instance, does a basic move substitution. In the event that the Caesar cipher is set for $\mathrm{A}=\mathrm{S}, \mathrm{B}=\mathrm{T}, \mathrm{C}=\mathrm{U}$, and so on.,

At that point, we can play out the accompanying encryption:

CAT $=>$ USL

One approach to accomplish dispersion is by muddling the images of a plaintext message. For instance, a transposition cipher is finished by composing the letters vertically one next to the other in segments of a settled tallness, and after that linking the columns through and through to shape the new message. The message "The crow flies at first light" can be composed in sections of stature 4 the accompanying way:

T R L A W

H O I T N

E W E D

C F S A

$\Rightarrow \quad$ TRLAWHOITNEWEDCFSA

Most cryptographic calculations include a blend of both confusion and diffusion.

Ayushi [4] clarifies Cryptography is the specialty of achieving security by encoding messages to make them non-significant. Cryptography is the practice and examination of covering information. In bleeding edge times cryptography is seen as a branch of both number-crunching and programming designing and is backup personally with information speculation, PC security and building. Cryptography is used as a piece of employments present in mechanically pushed social requests; delineations join the security of ATM cards, PC passwords and electronic business, which all depend on upon cryptography. There are two crucial sorts of cryptography: Symmetric Key and Asymmetric Key. Symmetric key estimations are the speediest and most customarily used kind of encryption. Here, a singular key is used for both encryption and disentangling. There are few comprehended symmetric key counts i.e. DES, RC2, RC4, IDEA etc. The maker portrays cryptography, diverse symmetric key figuring's in detail and after that proposes another symmetric key count.

\subsection{Symmetric Key Cryptography}

Symmetric key cryptography arrangements are all things considered requested as being either stream figure $\mathrm{s}$ or piece figure s. Stream figure s take a shot at a single square (byte or PC word) without a moment's delay, and complete some kind of feedback instrument so that the key is ceaselessly developing.

A square figure is asserted in light of the way that the arrangement scrambles one piece of data on the double using a comparative key on each piece. All things considered, the same plaintext piece will constantly encode to the same ciphertext while using an unclear key from a part of a square figure however the same plaintext will scramble to different ciphertext in a stream figure. Stream figures arrive in a couple enhances however two 
legitimacies indicating here. Self-synchronizing stream figure s processes each piece in the keystream as a component of the past $\mathrm{n}$ bits in the keystream. It is named "self-synchronizing" in light of the way that the unscrambling strategy can stay synchronized with the encryption system just by knowing how far into the n-bit keystream it is. Synchronous stream figure $s$ delivers the keystream in a way free of the message stream however by using the same keystream period work at sender and gatherer. While stream figure s doesn't spread transmission botches, they are, by their slant, discontinuous so that the keystream will at last repeat.

Square figure $\mathrm{s}$ can work in one of a couple of modes; the going with four are the most basic: ElectronicCodebook(ECB), Cipher-Block-Chaining(CBC), Cipher-Feedback(CFB) mode and Output-Feedback(OFB). The most generally perceived riddle key cryptography plot used today is the Data-Encryption-Standard(DES), illustrated by IBM in the 1970s and got by the National-Bureau-of-Standards(NBS) in 1977 for business and unclassified public supplications. DES has been gotten as Federal-Information-Processing-Standard-46(FIPS 46-3) and by the American-National-Standards-Institute as X3.92.

There are different other puzzle key cryptography estimations that are also being utilized today like CAST128(block-figure), RC2(block-figure) RC4(stream-figure), RC5 (block-figure), Blowfish (block-figure), Two fish (square figure).

\subsection{Motivation}

The paper closes with some proposed extensions to the OSI display with model's application to information security issues. What's more, some cryptographic arrangements: DES(block-figure), RC4(stream-figure), SHA1(hash), and RSA (open key cryptography) were upgraded.

This paper likewise tells symmetric-key stream cipher procedures and each one of these counts takes $n$ bits' plaintext as data and gives the extremely same number of bits as yield by using k bits' puzzle key.

\section{RELATED WORK}

In [5], the author gives data on different symmetric-key-cryptographic calculations to be examined for execution assessment, to choose the top calculation with fitting framework reasonable to give safety to information. Symmetric-key-cryptographic cipher s come in dual assortments, stream-cipher and block-cipher s. Streamcipher s deals with a flood of bits or bytes. Stream-cipher s are utilized for giving secure information of end stage and remote supplications. Block-cipher s performs enciphering or unscrambling on settled area of information. The plaintext is not generally in several of block-size, subsequently cushioning bits are expected to remunerate somewhat flow through block. The cushioning plan characterizes how the plaintext is loaded with information for final block. In system supplications block-cipher s are utilized for transfer of records of enormous lengths requires more security. Disentangling encrypted text without knowing the key is called cryptanalysis. Crypt-analysis of block-cipher $\mathrm{s}$ is troublesome contrasted with stream-cipher s. Consequently, in the vast majority of the supplications, block-cipher $\mathrm{s}$ is utilized for giving preferred safety over stream-cipher $\mathrm{s}$. Numerically connected arrangements of operations are utilized as parts of Substitution-change organize in encrypted manner to build the block of the Symmetric-key-cryptographic-block-cipher. In SP arrange key and plain content are taken as information and no of rotating circles of S-Box-substitution and change are connected to take a solitary ciphertext-block. The turnaround scheme is accomplished for decoding of the codes. Symmetric-key-cryptographic-cipher has distinctive steps and are utilized to build the block of the diverse Symmetric-key-block-cipher s. There are symmetric-key-structures like Feistel system, substitution-stage arrange and so on. On account of Fiestel system, the encryption and decoding procedure of the block are practically like each other, aside from it needs the inversion of key calendar. Cycle is a trademark highlight of Fiestel-system-cipher as an interior capacity knows as circular capacity.

In [6] the author thought about A-E-S and R-C-4 calculation and execution measurements were encryptionthroughput, CPU-work-stack, memory-usage, and key-size-variety and encryption and decoding time comes about demonstrate that the R-C-4 is quick and vitality putting something aside for encoding and decoding. R-C4 turned out to be superior to A-E-S for bigger length information.

Security is required to transmit characterized information over the framework. Security is also asking for in broad assortment of employments. Cryptographic figuring's accepting a key part in giving the data security against malignant assaults. In any case, then again, they devour huge measure of processing assets like CPU time, memory, encryption time and so forth. Regularly, symmetric-key-calculations are utilized over unbalanced-key-calculations as quick in environment. Symmetric-calculations are delegated block-cipher and stream-cipher s calculations. In this paper, we contrast the AES calculation and diverse methods (block-cipher) and R-C-4 calculation (stream-cipher) as far as CPU-time, encryption-time, memory-use and throughput at various stages like variable-key-size and variable-information-bundle-measure.

In [7] the author thought about cipher calculations (A-E-S, D-E-S, Blow-fish) for various ciphers-block-modes (E-C-B, C-B-C, C-F-B, O-F-B) on various document length fluctuating from $0.003 \mathrm{mb}$ to $0.203 \mathrm{mb}$. Blow-fish calculation executes better for all block-cipher-modes that were tried and O-F-B block-modes gives preferable execution over other block-modes. 
Security is the toppest difficult angles in web and system supplications. Web and systems supplications are developing quickly, so the hugeness and the estimation of the exchanged data over the web or other media sorts are growing. In this way, the output for the best response for offer the essential protection against the data intruders' strikes nearby giving these organizations in time is a champion among the most captivating subjects in the security related gatherings. Cryptography is the some of the fundamental classes of PC-security that proselyte's data from its typical shape into an indistinguishable frame. The dual principle qualities that recognize and separate an encrypted calculation from other are its capacity to safe the ensured information opposite to assaults and its rate and productivity in present as such.

The author gives a reasonable correlation via three most regular symmetric-key-cryptography calculations: D-ES, A-E-S, and Blow-fish. Since primary worry here is the execution of computations under different settings, the showed relationship contemplates the lead and the execution of the figuring when various data weights are used. The examination is made on the start of these parameters: speed, block-size, and key-size.

In [8] the author thought about cipher calculations (A-E-S, D-E-S, 3-D-E-S and Blow-fish) for fluctuating document estimate and looked at the encoding time on dual unique machines Pentium-4, 2.4 GHz and PentiumII $266 \mathrm{MHz}$ in E-B-C and C-F-B Mode.

The chief objective managing the outline of any encryption calculation must be security against unapproved assaults. In any case, for every single functional application, execution and the cost of usage are likewise essential concerns. An information encryption calculation would not be of much utilize in the event that it is sufficiently secure yet moderate in execution since it is a typical session to insert enciphering calculations in different supplications, for example, web based business, saving money, and online exchange handling applications. Implanting of encryption calculations in different applications likewise blocks an equipment execution, and is hence a noteworthy reason for debased general execution of the framework. In this paper, the four of the well-known mystery-key-encryption-calculations, i.e., D-E-S, 3-D-E-S, A-E-S (Rijndael), and the Blow-fish are being executed, and their execution is looked at by scrambling input documents of fluctuating substance and lengths, on various physical stages. The calculations are being actualized in a uni dialect, utilizing the standard details, to permit a reasonable correlation of running rates. The execution comes about have been compressed and a final overview has been introduced. In light of the investigations, it has been reasoned that the Blow-fish is the topmost performing calculation on the calculations decided for usage.

The authors in [9] exhibit a usage of 3 encryption-calculations and a correlation through them in light of CPUexecution-time. The CPU-execution-time is separated to bit and client-time. Chose calculations are: D-E-S, Triple-D-E-S (T-D-E-S) and Blow-fish. These are symmetric-block-encryption calculations. The target of this exploration is to assess the execution of the 3 cryptography calculations as far as the preparing time required in the block and client space for creating the mystery key, encryption and decoding operations. The intense versatile coding dialect Java-code and JCA (Java-crypto-analysis design) is utilized as a part of actualizing the encryption calculations. The execution of the actualized encryption calculations will be assessed on Sun-OS stages. The outcomes demonstrate that the Blow-fish calculation is the speediest, trailed by the D-E-S calculation then the T-D-E-S calculation.

In [10], A cryptographic calculation, or cipher, is the numerical limit used for encryption/unravelling. In case the security of a figuring relies on upon keeping it puzzle, it is a limited cipher. Limited cipher s is verifiably fascinating yet not sufficient now. Using a change in client group, data suffers in lost if the fake person finds the encrypted information. In addition, there is no quality control capacity to have on the calculation till the data should be covered up. Much more ideal are cipher s that depend on an openly known calculation that acknowledges a mystery-parameter, or key, for encoding and decoding. In the event that the encoding and unscrambling-keys are same (or numerically resultant with one another), the calculation is called symmetricalgorithm (D-E-S in this case):

$\mathrm{C}=\operatorname{Enc}(\mathrm{k}) \cdot \mathrm{M}$

$\mathrm{M}=\operatorname{Dec}(\mathrm{k}) \cdot \mathrm{C}$

On the off chance that the key used for encoding is not same as the key used for decoding, then the calculation is an open key algorithm (RSA is a case). The unscrambling key can't be computed from the encryption enter in a sensible measure of time (and the other way around). The reason it is known as an open key calculation is on the grounds that the encryption key can be made open. An outsider can in this manner scramble a text with this open-key yet just the receiver of the decoding-key (private-key) can unscramble the text. A text can likewise be encoded with the private-key and decoded with general public-key. This is utilized as a reason for advanced marks. Anybody can decode the text with the general public-key yet thusly, they realize that exclusive the holder of the private key could encode it (and subsequently made the text).

One of the filter of capacity that is key to open-key-cryptography is the restricted capacity. The capacity where it is generally simple to process $\mathrm{f}$.(x) however to a great degree hard to cipher $\mathrm{x}$, given $\mathrm{f}$.(x) (that is, the opposite capacity f.(x)). By to a great degree troublesome, we mean a many-sided quality that would take a large number 
of years if every one of the PCs on the planet were allotted to the issue. One frequently referred to state of mind around a restricted capacity is to consider breaking a plate. It's vastly less demanding to break the plate than it is to assemble the plate.

In [11], Data encryption is utilized inescapably as a part of today's associated society. The two most fundamental aspects of cutting edge information encryption are information security and validation. As presentday society turns out to be more associated, and more data gets to be distinctly accessible there is a requirement for shields which bring information honesty and information mystery. Likewise, confirming the wellspring of data gives the beneficiary, with finish sureness that the data original comes from first point and that it not been adjusted on its unique mode. The two requirements for data protection and data confirmation has persuaded crypto-techniques.

- Crypto-system or cipher-framework - The technique for camouflaging texts so that lone some individuals can see via mask.

- Crypto-graphy - The craft of making and utilizing crypto-systems.

- Crypt-analysis - The craft of removing crypto-systems, and see via camouflage notwithstanding where there is no capacity to do.

- Crypto-logy - The investigation of crypto-graphy and crypt-analysis.

- Plain-text - The first texts

- Cipher-text - The hidden text

- Encoding - A technique in which the normal information (plain-text) into ciphertext.

- Decryption-A technique to change over cipher-text into plain-text.

Encoding procedures are utilized to defend data till its put away inside a system hub or its in travel crosswise over interchanges media between hubs. A cryptosystem is normally an entire gathering of calculations. The calculations are marked; and the names are called keys. The general population who should have the capacity to see through the camouflage are called beneficiaries. Other individuals are adversaries, rivals, intruders, spies, or outsiders.

For instance, for a plain-text info is sent, if each An is supplanted with a ' $D$ ', each ' $B$ ' is supplanted with an ' $E$ ', thus on through the letters in order, just somebody who knows the "move-by-3" manage can interpret the texts. Henceforth a "move-by-n" encoding method can be showed for few unique estimations of $n$. along these lines, $n$ is the key here.

With the extension of utilizations requiring information encryption, the quantity of various scrambling techniques has additionally expanded. Every strategy has its qualities and shortcomings. A portion of the encryption strategies are R-S-A (Rivest-Shamir-Adleman), Data-Encryption-Standard (D-E-S), Diffie-Hellman, Secure-Hashing-Algorithm (S-H-A), Blow-fish, R-C-4/R-C-5, Elliptic-Curves, El-Gamal, L-U-C (LucasSequence) etc.

The author in [12], tells about the Data-Encryption-Standard (D-E-S) is block-cipher which considers a settled size of plain-text minutes and change via progression of entangled methods into another cipher-text bit-string of a similar size. It is a symmetric-encryption system which implies both mailer and recipient use a shared-key to scramble and additionally decode the information as appeared in the underneath Fig 3:

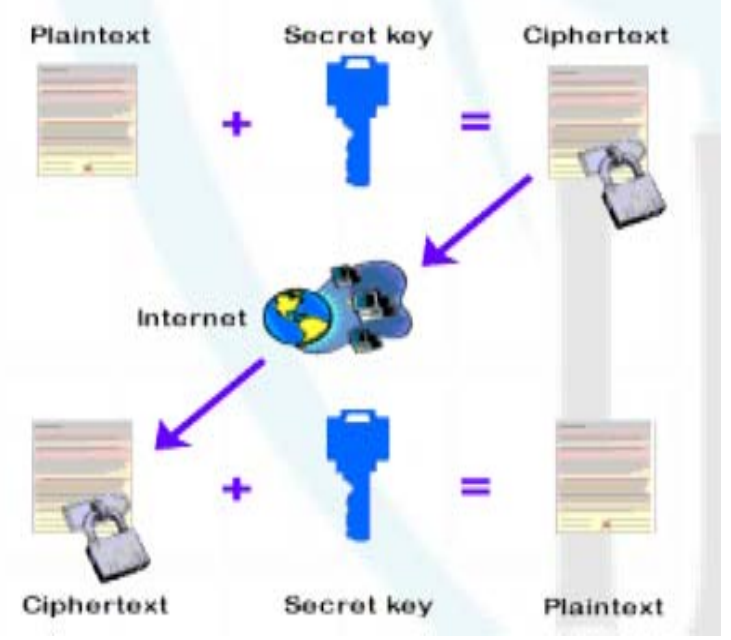

Fig 3: Conversion of Secret key 
In [13], Wireless systems assume basic parts in present work, home, and open spots, so the requirements of securing of such systems are expanded. Encryption calculations assume imperative parts in data frameworks security. Those calculations devour a lot of registering assets, for example, CPU time, memory, and battery control. CPU and memory convenience are expanding with a reasonable rate, yet battery innovation is expanding at slower rate. The issue of the slower expanding battery innovation shapes "battery hole". The outline of effective secure conventions for remote gadgets from the perspective of battery utilization needs to see how encryption strategies influence the utilization of battery power with and without information transmission. This paper concentrates the impacts of six of the most widely recognized symmetric encryption calculations on power utilization for remote gadgets. at various settings for every calculation. These setting incorporate distinctive sizes of information blocks, diverse information sorts (content, pictures, and sound record), battery control utilization, diverse key size, diverse instances of transmission of the information, impact of differing sign to commotion proportion lastly encryption/decoding speed.

Authors in [14] discloses differences via mystery key and open-key-cryptography with existing work, for encoding and decoding there are dual viewpoints: calculation for key utilized for encoding and unscrambling. Key is like single time cushion utilized as a part of vernam cipher. On the off chance that similar key is utilized for encoding and unscrambling and then it is called symmetric-key-cryptography. What's more, if distinctive keys are utilized for encryption and unscrambling this lopsided key-cryptography. In symmetrical-keycryptography one key is utilized. So as before dispersing the information between elements the key must be exchanged. Symmetric-key-cryptography tells D-E-S, A-E-S, 3-D-E-S, Blow-fish computations and etc and hilter kilter key-cryptography incorporates R-S-A, Digital-Signature and Message-Digest-calculations.

\section{Parity Concepts}

Benny Applebaum et al [15] attempt to expand the establishments of open-key-cryptography. Creators develop new open-key-encryption plans in light of new hardness by and large suspicions for common combinatorial NPhard improvement issues.

Creators consider the accompanying suspicions:

1. It is infeasible to tackle an irregular arrangement of inadequate direct conditions mod 2, of which a little division is boisterous.

2. It is infeasible to recognize an irregular unequal bipartite diagram, and such a chart in which we "plant" at arbitrary in the huge side a set $\mathrm{S}$ with just $|\mathrm{S}|=3$ neighbours.

3. There is a pseudorandom generator in $\mathrm{NC} 0$ where each yield relies on upon an arbitrary steady size subset of the data sources.

Creators get semantically secure open key encryption plans in view of a few mixes of these suppositions with various parameters. Specifically, creators get open key encryption from Assumption 1 all alone, yielding the main loud conditions sort open key plan in which the commotion rate is higher than one over the piece foundation of conditions. Creators likewise get open key encryption in view of a blend of Assumptions 2 and 3. These are ostensibly of more "combinatorial"/"private-key" nature than any suppositions utilized before for open key cryptography. Creators confirmation includes novel "inquiry to choice" and "pursuit to expectation" diminishments for scanty boisterous straight conditions.

The quality of creator's suspicions raise new algorithmic and pseudo irregularity questions (and new parameters for old ones). Creators give some proof for these suspicions by concentrate their imperviousness to specific classes of regular calculations, including semi-distinct projects, AC0 circuits, low-degree polynomials, and cycle numbering. Creators likewise relate our presumptions to different issues, for example, planted inner circle and learning juntas.

Chris Peikert [16] builds open-key-cryptosystems that are secure accepting the most pessimistic scenario hardness of approximating the base separation of n-dimensional cross sections to inside little poly (n) elements. Earlier cryptosystems with most pessimistic scenario associations were construct either in light of the briefest vector issue for an exceptional class of cross sections, or on the guessed hardness of grid issues for quantum calculations.

Creator's primary specialized development is a lessening from variations of the most limited vector issue to relating variants of the "learning-with-errors" (LWE) issue; beforehand, just a quantum decrease of this kind was known. As an extra commitment, we build a characteristic picked cipher-text-secure crypto-system having a much more straightforward depiction and more tightly basic most pessimistic scenario guess consider than earlier plans in light of cross sections.

A few scientists introduced a review of symmetric-block-cipher calculations as E. Surya et al (2012) [17] presented a nitty gritty overview on symmetric-key-block-cipher calculations. The analyst clarifies the necessities of safety as protection, uprightness, verification, non-disavowal and get to control and gives the significance of each of them in the crypto-graphy. This paper focused on introduced brief definitions for most 
normal encoding computations as indicated by ordered it in crypto-graphy where the scientists grouped calculations in this into symmetric-calculations which utilizes one a mutual key amongst sender and beneficiary, for example, D-E-S, A-E-S, triple-D-E-S and Blow-fish, and hilter kilter calculations (open key calculations) which utilizes two distinctive keys, for example, RSA. After looked at all encryption techniques specified over the scientists demonstrate that Blowfish calculation utilizes 32-bits to 448-bits variable number and the information can encrypt to16 times.

Lars R Knudsen et al (1998) [18] outline a review on block-cipher-key where the scientists focus on the fundamental utilization of block-cipher s and the best in class of cryptanalysis of block-cipher s. The specialists amid this review disclose an approach to break numerous frameworks snappier than by a comprehensive look for the key.

\section{SYMMETRIC-KEY-BLOCK-CIPHER}

Symmetric-key-block-cipher are comprised of 2 calculations E (Encryption) and D (Decryption) and every one of these calculations takes $n$ bits of plain-text as info and provides the very similar no of bits as yield by utilizing $\mathrm{k}$ bit’s mystery-key. Block-cipher can be delegated appeared in accompanying fig 4 .

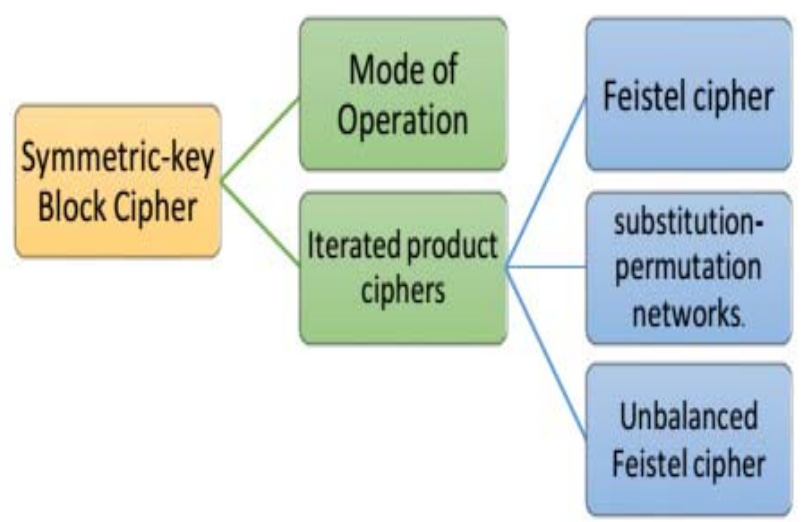

Fig 4: Symmetric-Key Block Cipher categories

\subsection{Mode of Operation}

Method of operation methods for utilizing block-cipher for encoding used to apply block-ciphers to bigger plain-texts. Method of operation grouped into deterministic and probabilistic appeared in fig underneath

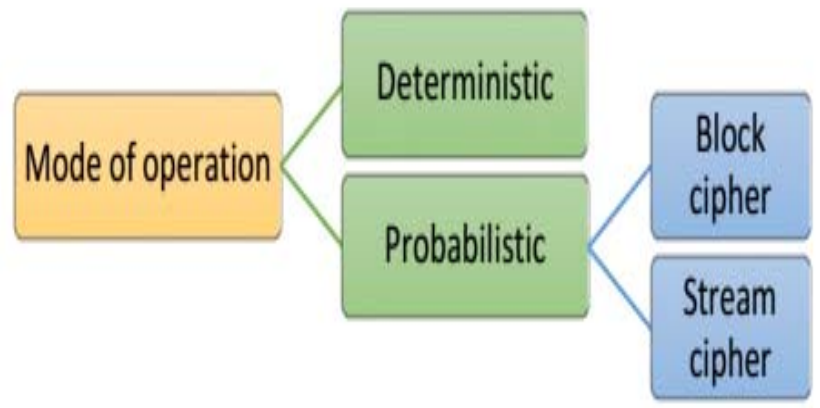

Fig 5: Mode of operation category

In incorporation to the above classification, a few block-cipher methods of working intended for mystery and confirmation in a solitary crypto-graphic primitive. Paper portray small outline for a wide range of formats exhibited by analysts and this tells the cause. The Electronic-Code-Book (E-C-B) is destinism method of working the most widely recognized path in figuring texts. This format partitioned texts into no of blocks and encodes every block in isolated frame from one another. The primary preferred stands of this mode, is that the setting among encoding and decoding is a bit much, for this situation when the collector not got all encoded blocks in light of the fact that happen issues in transmission prepare, the beneficiary can just unscramble the got obstructs with no issue. ECB mode gives rapid in execution in light of the fact that E-C-B mode work alternative to the figuring process [19].

The Cipher-Block-Chaining (C-B-C) mode and the Cipher-Feed-back (C-F-B) mode were make the modes of cipher-content reliant on the all past blocks of plain content through figuring process [20]. 
Dark and P Rogaway (2002) [21] characterize another method of working said P-M-A. The new P-M-A-C mode utilized for text confirmation. P-M-A-C is passivity bolstered every block size of strings, where utilizes single key and this $\max \{1,[|\mathrm{M}| / \mathrm{n} \mid\}$ block-cipher calls to M-A-C a string $\mathrm{M} \in\{0,1 \mid\}$ utilizing a n-bit block-cipher.

$P$ Rogaway et al [22] characterizes also with portray another similarly block-cipher method of working which gives twain classification and validation in meantime, the new way said O-C-B mode "balance-codebook". O-C$B$ encodes string $M \in\{0,1\}$ utilizing $[|M| / n]+2$ block-cipher where $n$ is the block-size. The fundamental components of O-C-B mode is capricious length messages and insignificant size cipher writings, Nearly ideal number of block-cipher calls, no prerequisite for an irregular IV, well organized counterbalance computations and Union basic key.

M Bellar et al [23] displayed another method of working called E-A-X. This proposed to take care of the issue connected with A-E-A-D (verified encryption with related information), and E-A-X described that it's on the web, settled top head, and successful for evacuating the cost of pre-message to cipher-text.

Morris J Dwork [24] exhibited his work characterize another method of working which gives proposal for Block-Cipher method of working. The new mode said C-C-M mode for symmetric-key-block-cipher. The mode joining the systems of twain modes, the counter-mode and C-B-C-M-A-C calculation, C-C-M used for protection also with realness of information.

$\mathrm{T}$ Kohno et al [25] present another mode said C-W-C for securing both the privacy and the verification of delicate data. C-W-C having numerous qualities verifiable security, similar way of ability, elite equipment with programming bundle, and no licensed innovation approach. In view of these qualities $\mathrm{C}-\mathrm{W}-\mathrm{C}$ an effective device for use in a few execution basic supplications, C-W-C can prepare information at $10 \mathrm{Gbps}$ in equipment.

S Halevi et al [26] delineates another way for block-cipher called E-M-E where changing over n-bit blockcipher into $\mathrm{m}$-n-bits (where $\mathrm{m}$ has a place with $[1 \ldots \mathrm{n}]$ ) a two-way encoding conspires that chips away at strings. The new mode E-M-E is proficient and simialarable that used to take care of the issue of plate area encoding. The analysts demonstrated E-M-E is secured for advanced crypto-graphy.

David A N et al [27] presented another mode for block-cipher called Galois or Counter Mode (G-C-M) for encoding and text verification. Galois or Counter (G-C-M) method of working has qualities where function as a quality a solitary text validation program and acknowledge $4 \mathrm{~s}$ of self-assertive size additionally the specialists demonstrate the G-C-M is secure in the quality model of security.

Tetsu Iwata [28] introduced another method of working in block-cipher called C-E-N-C. C-E-N-C is Cipherbased Encoding. C-E-N-C is described by more components as exceedingly effective, union key, irregular get to and so forth.

C Jutla [29] characterize another method of working called Integrity-Aware-Parallelizable-Mode (I-A-P-M) for block-cipher s. This new method gives tweak security and respectability of texta and very simialarable.

M J O Saarinen [30] displayed another confirmed block-cipher method of working called Sophie-GermainCounter-Mode (S-G-C-M). This method utilized with 128-block block-cipher s, for example, A-E-S. S-G-C-M is vary from Galois or Counter Mode where S-G-C-M utilize G F (p) with $\mathrm{p}=2128+12451$, where (p-1)/2 is additionally a single, to a great extent in fact perfect other option to GCM. And in addition to above methods there is a few methods intended to transforms a block-cipher into a stream-cipher s, for example, Cipher-Feedback (C-F-B), Output-Feed-back (O-F-B) and Counter(CTR).

\subsection{Iterated Product Ciphers}

In 1949, the Shannon's proposed the idea of item-cipher, Shannon's characterize tweak working of a safe Cipher perplexity which imply that the connection between the normal content and encrypted content is extremely mind boggling, For instance temporary fill-up table. What's more, dispersion which means the impact of single block from normal content is a spread over many encrypted content bits. In instance, stage. Join Confusion and dispersion ordinarily to fabricate a solid block-cipher and it called as item-cipher [31]. Various numerous acknowledge of block-cipher actualize as per the idea of item-cipher can be delegated Feistel-cipher and temporary stage systems and so on.

\subsubsection{Feistel-cipher: Introduction}

Feistel-cipher is a standout amongst the very most widely recognized figures in queued item cipher s utilizes by adding circular capacity loopely on the normal content to gives encrypted content with diffusion and perplexity qualities Encryption and decoding in the Feistel-cipher is comparative, one of the block-cipher calculations utilize the plan is Data-Encryption-Standard (D-E-S) [32] [33].

\subsubsection{Data-Encryption-Standard (D-E-S)}

D-E-S is Data-Encryption-standard plan by IBM and tells off a standout amongst the top vital calculations, distributed by NIST and turns into a quality in 1974. 
D-E-S structure comprises of 64-bits input normal content and 64-bits yield encrypted messages and upheld 56bits key-length [34]. Because the Encryption is procedure to guarantee the safety of transmission information over a shaky path, and as it is known remote channels it is an open way to gatecrashers and assaults, and in light of the fact that the figuring calculations used to scramble information in remote systems don't consider the bit mistake qualities of the remote channels.

Zibdeh et al. (2011) [35] exhibited another altered DES calculations (information encryption standard) to make it secure to the bit blunders brought on by the remote systems. The adjusted calculation enhances the bit mistake rate (BER) execution and also security contrasted with DES.

As the time need for crypt-analysis D-E-S has decreased and the equipment procedures grow rapidly, DES might be endangered to assaulted utilizing other way process, for this, Seung-Jo-Han et-al in Sept of 1996 propose change into Data-Encryption-Standard (D-E-S) calculation to guarantee the continual process of secure cryptography. The analysts introduced new plan of a D-E-S called Improved-D-E-S. Another calculation forms information blocks of lengths 96-bits where isolated the information obstruct into 3 sub blocks of size 32-bits, increment s-confines to 16 boxes (S1-S16) and bolstered key-length of size 112-bits, the enhanced calculation fulfilling the strict-Torrential slide standard (S-A-C) and co-relation co-efficient. The analysts demonstrate that the Improved-D-E-S is more grounded than the D-E-S against differential-crypt-analysis for crypto-graphic security and in this way the uni Diffuse (U-D) inside the Improved-D-E-S is expanded more than D-E-S's U-D [36].

\subsubsection{Feistel-cipher: Survey}

Notwithstanding the DES calculation said in the past area, a few block-cipher techniques are introduced in the field of Feistel-cipher-structure. In this area, a small diagram for some Feistel-cipher techniques advertised. Xiao-Jun-Tong et-al [37] introduced another encryption calculation for remote sensor arrange in light of compound clamorous guide and Feistel organize. The new technique is block-cipher-key develops a cubical capacity including discretisized riotous guide. The analysts demonstrate that another combined disorganized block-cipher appropriate for the remote-sensor-systems, since another block-cipher has more secure and effectiveness, less asset consumption and this show up via trial of security and execution of new encodingtechnique.

Rashmi et-al [38] exhibited another calculation to enhance the encryption proficiency of the current RC6 calculation said R-C-7. R-C-7 does utilization of six operating registers rather than four uses in R-C-6 and It has a block-size of 256 bits. The new calculation sets aside less opportunity to scramble information and it is much more adaptable.

B Schneier et-al [39] exhibited another encryption calculation of block-cipher-key called Blow-fish, another block-cipher Blow-fish forms information mode of size 64-bits and bolstered variable key-length up to 448-bits. Blow-fish in view of Feistel-cipher where including sixteen-round and every single round comprises from dual sections key stages and key-information-substitution. This calculation reasonable for supplication that won't change key since Blow-fish calculation not fulfilling every one of the prerequisites for cutting edge cryptographic but rather it is seem quicker than D-E-S (Data-Encryption-Standard) when executed on 32-bit microchips with bigger information stores.

Schneier et al. (1998) [40] introduced another block cipher in light of Feistel system comprises of sixteen-round with Fish work called TWO-FISH. TWO-FISH-encryption calculation forms 128-bits block-measure and upheld key-length up to 256-bits. The fresh calculation can execute in equipment in 14000 entryways. TWOFISH calculation intended to fulfil N-I-S-T outline criteria for A-E-S (Advanced-Encryption-Standard). The fundamental components or properties of TWO-FISH that it is symmetric-key-block-cipher, proficiency in tweak programming and equipment for few stages. Straightforward and adaptable plan, easy usage and suitable for a stream-cipher, hash-capacity and MAC.

Ronald L R et-al [41] displayed the characterize and portray a quick symmetric-block-cipher-key called R-C-5 encoding calculation where utilized an indistinguishable key from a part of tweakable encoding and unscrambling. R-C-5 calculation variable in each of word-size, number-of-rounds and key-length, this calculation utilized just calculational primitive working and hence R-C-5 calculation ought to be suitable for equipment or programming.

Akihiro Shimizu et-al [42] exhibited another block-cipher calculation for safer correspondences and collected area information called F-E-A-L. F-E-A-L is documentation of Fast-Data-Encipherment-Algorithm with blocksize 64-bit and 64-bit key-length. F-E-A-L like D-E-S in secured information and it is reasonable for programming and equipment executions as D-E-S. 


\subsubsection{Substitution-Permutation-Networks (S-P-N): Introduction}

Substitution-Permutation-network is another structure in iterative item cipher s. substitution-change organize initially presented by Feistel et-al [43] [44] alluded to as S-P-N. S-P-N is arrangement of scientific workings utilized as a part of block cipher. Substitution-stage organizes comprising of a succession of substitution round box said S-boxes and associated by particle position changes or Reverse Alternative.

\subsubsection{Advanced-Encryption-Standard (A-E-S)}

A-E-S is the most broadly utilized symmetric-cipher. N-I-S-T Calls A-E-S in 1997, A-E-S block-cipher with 128-block block-size and incorporate 3 key-lengths more likely than not upheld 128, 192 and 256 blocks. A-E-S block-cipher is effectiveness in programming and equipment, a few industry and business frameworks incorporate A-E-S, for example, Internet-security-standard IP_sec, IEEE 802.1, S-S-H (secure-shell), and so forth [45].

In [46] October 2, 2000 Rijndael et-al calculation comprises of 3 stages beginning-round called Add-Round-Key, Standard-Round comprises of 4 changes: Sub-Byte, Shift-Row, Mix-Column and Add-Round-Key, and the last round likewise comprises of Sub-Byte, Shift-Row and Add-Round-Key however excluding the Mix-Column change. In general execution, in light of velocity of encoding / decoding procedure and key-set-up-time, this calculation can apply in a few supplications, for example, brilliant-cards and different supplications which expected to put away and shielding delicate data from unapproved get to.

Hanem et al. (2012) [47] displayed another approach by changed S-boxes and enter development system in cutting-edge-encryption-standard called Modified-Rijndael-Algorithm (M-R-A) where planning little s-boxes characterized over G-F (24) instead of G-F (28). The adjusted Rijndael calculation accomplishes confusion, diffusion and high-security. From the execution assessment of adjusted Rijndael calculation demonstrate that M$\mathrm{R}-\mathrm{A}$ is more appropriate for the supplications that requires high-security.

Iqtadar et-al [48] exhibit another encryption strategy in view of another S-8 S-Boxes to build 4032040320 mystery keys. The new-approach more frameworks secure comprises of 2 gatherings to accomplish safe correspondence channel, the trading of mystery texts uses $n 40320$ key alternatives and the sender of the correspondence session need to change key with every message of size 16 for this another encoding technique gives more safe frameworks on the grounds that if interlopers endeavour to cut the code for these framework expected to see all $n 40320$ keys or watches the letter set recurrence of figuring message. The new encryption technique gives secure many-sided quality as A-E-S (Advanced-Encryption-Standard).

\subsubsection{SPN: Survey}

A few substitution-stage systems and block-cipher techniques were said by specialists. This area we will be in favour of some of this technique and tells small review about every strategy. Kazys et-al [49] introduced another approach for outlining key-subordinate S-Boxes and reverse S-Boxes era calculation for block-cipher-systems where altering just a single block of key to create key ward S-Boxes.

Hong jun et-al [50] exhibited another block-cipher calculation for verified encoding techniques called A-E-G-IS. A-E-G-I-S used to ensure information and system bundles. The new calculation accomplishes speed 7/10 clock cycles/bytes for 4046 byte-messages. A-E-G-I-S 128 utilizations 5 round-capacity of A-E-S and A-E-S256 utilizations 6 round-capacity.

Jian-Guo et-al [51] introduced about another block-cipher calculation called L-E-D calculation. This calculation intends to accomplish and stay execution user's data for programming usage furthermore LED block cipher devoted to work in equipment execution.

Daesung et al. (2003) [52] introduced paper offers another encryption calculation for block-cipher called A-R-IA. A-R-I-A forms information block-of-size 128-bits and in light of substitution-stage organize system of iterative item cipher s. A-R-I-A utilizes just essential working where utilize s-encloses the similar utilizations the Rijndael calculation and in addition XOR operation with the goal that it is proficient executions for different situations (reasonable for various stages).

Joan et al. (1997) [53] exhibited another encryption calculation for block cipher frameworks called BLOCK. Another calculation in light of substitution-stage-cipher and procedures-128-block block-size and upheld 128block key-length. BLOCK block-cipher calculation planned over straight and differential-assaults. BLOCK apply in a few touchy supplications where it is proficient equipment usage and has high-mutualism.

Many techniques join S-P-N and Feistel-Cipher-structure from this strategy Takeshi et-al [54] displayed another encryption calculation for symmetric-block-cipher called SC-2000. The new block-cipher tells in light of two Fiestel-cipher and substitution-change-cipher. The new SC-2000 Symmetric-block-cipher calculation gives fast to both programming and equipment usage for various stages and accomplishes abnormal state of figuring security. 
Eli Biham et-al [55] exhibited and offers another calculation for block-cipher to fulfilling Advanced-encryptionstandard (A-E-S) prerequisites called Ser-pent. Ser-pent bolstered block-size of 128-block and 256-block keylength. The new calculation is an exceptionally proficient usage and high secure against a wide range of assault.

\subsubsection{Unbalanced Feistel-cipher: Survey}

Generalized-Unbalanced-Feistel-Network(G-U-F-N) recommended by Schnedier et-al [56] tantamount to routine Fiestel-cipher, Unbalanced-Fiestel-cipher incorporate a succession of circles where the block is isolated into 2 sections not equivalent in sizes, this change on Fiestel-cipher has fascinating ramifications for planning cipher s secure over direct and indirect assaults.

A few block cipher s has been built in light of Unbalanced Feistel Network where TaizoShirai et al. (2007) [57] introduced another encryption calculation for block cipher frameworks called CLEFIA the new calculation in view of lopsided Feistel-cipher and perfect with A-E-S.

Deuki Hong et-al [58] exhibited another encryption technique for symmetric-block-cipher in view of Generalized-Unbalanced-Feistel-Network called H-I-G-H-T utilized for less assets gadgets and procedures information-block of size 64-bits and bolstered key-length of size 128-block. To achieve security, we demonstrate the new calculation H-I-G-H-T block-cipher has enough safety for touchy data over assaults.

Matt Blaze et-al [59] displayed another block cipher in view of uneven Feistel-cipher in which each way of the ciphers alters just sixteen bits for a capacity. Mac_Guffin like D-E-S encipher calculation in numerous qualities as block-size, application-area, execution and usage point, the block-cipher incorporates 32-round and upheld 64-bits block-size and 128-block-size of key.

\subsection{RC4: Survey}

Poonam Jindal et al [60] reviews the most pervasive and monetarily utilized RC4 stream figure alongside the counter_measures are exhibited in this survey work. Creators have concentrated on R-C-4 on the grounds that it beats among all the current stream figures. In spite of the fact that the calculation is openly uncovered in 1994 through web yet because of its plan straightforwardness everybody gets pulled in towards it and has been received around the world. The figure is broadly received in different programming and web applications. It is utilized as a part of various system conventions, for example, WEP (Wireless proportionate protection), WPA (Wi-Fi ensured get to), and S-S-L (Secure-attachment-layer). Additionally, this survey is broadly utilized as a part of Microsoft-windows, Apple-O-C-E (Apple-Open-Collaboration-Environment), secure-SQL (a server for database administration and information warehousing arrangement) and so on. All through the paper creators have attempted to investigate the different shortcomings of the figure till date. It is found that paying little mind to numerous endeavours done through analysts in enhancing the blemishes of R-C-4 figure, still there are no of predispositions occurs in the key-stream, key recuperation can be produced using state and some arrangements of keys do exist that can create comparative states. It verifies the way that after many years of survey the R-C-4 stream-cipher keeps on offering research issues important to specialists.

\section{CONCLUSION}

This paper made a considerable measure of progress in the environment of the O-S-I 7-Layer-Model and its utilization as a data safety device. Having broadened it with the consideration of clients and the approach they work under which many faculties secured the whole range of information affirmation. In either way of the standard system setting and in the expanded setting of data security, the 7-layer-model is best connected as a device for arranging ideas and situations instead of as a theoretical straight-jacket. Cases from both universes demonstrate that exemptions and differences to the model are some of the time alluring. Furthermore, survey gives small diagram on cryptographic-block-cipher strategies as per classifications in crypto-graphy. Survey gives an investigation of the encipherement calculation proposals in this work as per order it in crypto-graphy, for example, D-E-S, BLOW-FISH, TWO-FISH, and so on., arranged in Feistel Network, AES, AEGIS, ARIA, and so on., grouped in substitution-change systems and CLEFIA, HIGHT, MacGuffin, and so forth., characterized in Generalized Unbalanced Feistel Network (GUFN).

\section{REFERENCES}

[1] Damon Reed,” Applying the OSI Seven Layer Network Model To Information Security” November 21, 2003 , SANS Institute 2004.

[2] Kari A. Pace, "A Layered Security Model: OSI and Information Security”, Global Information Assurance Certification Paper, Copyright SANS Institute.

[3] Shelley Kandola, “A Survey of Cryptographic Algorithms”, May 13, 2013

[4] Ayushi, “A Symmetric Key Cryptographic Algorithm”, (C2010 International Journal of Computer Applications (0975 - 8887) Volume 1 - No. 15.

[5] Ranjeet Masram, Vivek Shahare, Jibi Abraham, Rajni Moona, “Analysis and comparison of symmetric key cryptographic algorithms based on various file features”, International Journal of Network Security \& Its Applications (IJNSA), Vol.6, No.4, July 2014

[6] Nidhi Singhal and J.P.S.Raina, "Comparative Analysis of AES and RC4 Algorithms for Better Utilization", International Journal of Computer Trends and Technology, Vol 2, Issue 6, July-Aug 2011, pp.177-181.

[7] Jawahar Thakur and Nagesh Kumar, "DES, AES and Blowfish: Symmetric Key Cryptography Algorithms Simulation Based Performance Analysis”, International Journal of Emerging Technology and Advanced Engineering, Vol 1, Issue 2, December 2011, pp.6-12. 
[8] Aamer Nadeem, Dr M. Younus Javed, “A Performance Comparison of Data Encryption Algorithms", First International Conference on IEEE Information and Communication Technologies (ICICT), Vol 1, Issue 6, 27-28 Aug. 2005, pp 84-89.

[9] Kofahi, N.A, Turki Al-Somani, Khalid Al-Zamil, "Performance evaluation of three Encryption/Decryption Algorithms", IEEE 46th Midwest Symposium on Circuits and Systems, Vol 2, Issue 1, 30-30 Dec. 2003, pp. 790-793.

[10] Paul Krzyzanowski, “Cryptographic communication and authentication”, Rutgers University - CS 417: Distributed Systems

[11] Amit Dhir, "Data Encryption using DES/Triple-DES Functionality in Spartan-II FPGAs", WP115 (v1.0) March 9, 2000, http://www.xilinx.com/legal.htm

[12] Karthik .S, Muruganandam.A, "Data Encryption and Decryption by Using Triple DES and Performance Analysis of Crypto System" International Journal of Scientific Engineering and Research (IJSER), Volume 2 Issue 11, November 2014.

[13] Abdul kader, Diaasalama and Mohiv Hadhoud, "Studying the Effect of Most Common Encryption Algorithms," International Arab Journal of e-technology, Vol.2. No.1.

[14] Aman Kumar, Sudesh Jakhar, Sunil Maakar, "Distinction between Secret key and Public key Cryptography with existing Glitches”, Volume: 1, 2012.

[15] Benny Applebaum, Boaz Barak, Avi Wigderson, “Public-Key Cryptography from Different Assumptions”, Institute for Advanced Study, Princeton, November 7, 2009.

[16] Chris Peikert, "Public-Key Cryptosystems from the Worst-Case Shortest Vector Problem”, Georgia Institute of Technology, September 1, 2009.

[17] E Surya, C.Diviya, “A Survey on Symmetric Key Encryption Algorithms”, International Journal of Computer Science \& Communication Networks, Vol 2(4), 475-477, 2012

[18] Lars R. Knudsen, "Block Ciphers -A Survey”, Springer Berlin Heidelberg, State of the Art in Applied Cryptography, Lecture Notes in Computer Science Volume 1528, 1998, pp. 18-48, 1998

[19] C. Paar and Pelzl, Jan, “Understanding Cryptography, A textbook for students and Practitioners”,Copyright Springer-Verlag , pp. 125

[20] Eli Biham, “On modes of operation”, Fast Software Encryption, Lecture Notes in Computer Science Volume 809, pp. 116-120, 1994

[21] John Black and Phillip Rogaway, “A Block-Cipher Mode of Operation for Parallelizable Message Authentication”, Springer Berlin Heidelberg, Advances in Cryptology —EUROCRYPT 2002, Lecture Notes in Computer Science Vol. 2332, pp. 384-397, 2002

[22] P. Rogaway, MihirBellare, John Black, “OCB: A blockcipher mode of operation for efficient authenticated encryption”,ACM Transactions on Information and System Security (TISSEC), Vol. 6, Issue 3,pp. 365-403, August 2003

[23] M Bellare, P Rogaway, D Wagner, "The EAX Mode of Operation”, Springer Berlin Heidelberg, Fast Software Encryption, Lecture Notes in Computer Science Volume 3017, pp 389-407,2004

[24] Morris J. Dwork, "SP 800-38C. Recommendation for Block Cipher Modes of Operation: the CCM Mode for Authentication and Confidentiality", National Institute of Standards \& Technology Gaithersburg, MD, United States (C2004.

[25] T. Kohno, J. Viega, D. Whiting, “CWC: A HighPerformance Conventional Authenticated Encryption Mode”, Springer Berlin Heidelberg, Fast Software Encryption, Lecture Notes in Computer Science Vol. 3017, pp. 408-426, 2004

[26] Shai Halevi and Phillip Rogaway, "A Parallelizable Enciphering Mode”,Springer Berlin Heidelberg, Topics in Cryptology - CTRSA 2004, Lecture Notes in Computer Science Vol. 2964, pp. 292-304, 2004

[27] David A. McGrew, John Viega, "The Security and Performance of the Galois/Counter Mode (GCM) of Operation",Springer Berlin Heidelberg, Progress in Cryptology - INDOCRYPT 2004, Lecture Notes in Computer Science Volume 3348, pp. 343-355, 2005

[28] Tetsu Iwata, "New Blockcipher Modes of Operation with Beyond the Birthday Bound Security", Springer Berlin Heidelberg, Fast Software Encryption, Lecture Notes in Computer Science Volume 4047, pp. 310-327, 2006

[29] Charanjit S. Jutla, "Encryption Modes with Almost Free Message Integrity", Springer-Verlag, Vol. 21, Issue 4, pp. 547-578, October 2008.

[30] MJO Saarinen, “SGCM: The Sophie Germain Counter Mode”, IACR Cryptology ePrint Archive, 2011.

[31] C. E. Shannon, "Communication Theory of Secrecy Systems*”, Bell System Technical Journal, Vol. 28, Issue 4, pp. 656-715, October 1949

[32] Kaisa Nyberg, “Generalized Feistel networks “, Springer Berlin Heidelberg, Advances in Cryptology —ASIACRYPT '96, Lecture Notes in Computer Science Volume 1163, pp. 91-104, 1996

[33] Lars R. Knudsen, "Practically secure Feistel ciphers", Springer Berlin Heidelberg, Fast Software Encryption, Lecture Notes in Computer Science Volume 809, pp. 211-221, 1994

[34] Jawahar Thakur, Nagesh Kumar, "DES, AES and Blowfish: Symmetric key cryptography algorithms simulation based performance analysis”, International Journal of Emerging Technology and Advanced Engineering, ISSN 2250-2459, Volume 1, Issue 2, December 2011.

[35] Zibideh, W.Y.; Matalgah, Mustafa M. "Modified DES encryption algorithm with improved BER performance in wireless communication,” Radio and Wireless Symposium (RWS), 2011 IEEE, pp.219-222, Jan. 2011

[36] Seung-Jo Han; Heang-Soo Oh; Jongan Park, "The improved data encryption standard (DES) algorithm”, Spread Spectrum Techniques and Applications Proceedings, IEEE 4th International Symposium on (Volume:3),1996.

[37] Xiao-Jun Tong; Zhu Wang; Yang Liu; Miao Zhang; Lianjie Xu, "A novel compound chaotic block cipher for wireless sensor networks," communications in Nonlinear Science and Numerical Simulation vol.22. Issues.1-3, May 2015, pages 120-133

[38] Rashmi; Chawla, Vicky; Nagpal, Rajni Sehgal Renuka,” The RC7 Encryption Algorithm” International Journal of Security \& Its Applications. 2015, Vol. 9 Issue 5, p55-59. 5p.

[39] Bruce Schneier,” Description of a new variablelength key, 64-bit block cipher (Blowfish)”, Springer Berlin Heidelberg, Fast Software Encryption, Vol 809 of the series Lecture Notes in Computer Science pp 191-204, 2005

[40] B Schneier, J Kelsey, D Whiting, D Wagne, Chris Hall, Niels Ferguson, “Twofish: A 128-bit block cipher” -NIST AES 1998 - repo.hackerzvoice.net

[41] Ronald L. Rivest "The RC5 encryption algorithm", Springer Berlin Heidelberg, Fast Software Encryption, Lecture Notes in Computer Science Volume 1008, 1995, pp. 86-96

[42] Akihiro Shimizu, Shoji Miyaguchi "Fast Data Encipherment Algorithm FEAL" Springer Berlin Heidelberg, Advances in Cryptology - EUROCRYPT' 87, Lecture Notes in Computer Science Volume 304, 1988, pp. 267-278

[43] Howard M. Heys, Stafford E. Tavares,"Substitutionpermutation networks resistant to differential and linear cryptanalysis”, Journal of Cryptology, March 1996, Volume 9, Issue 1, pp. 1-19

[44] H. Feistel, W. A. Notz, and J. L. Smith. Some cryptographic techniques for machine-to-machine datacommunications. Proceedings of the IEEE, Vol.63 (Issue 11): pp.1545-1554, 1975 
[45] Paar, Christof, Pelzl, Jan, “The Advanced Encryption Standard (AES)” Understanding Cryptography, A textbook for students and Practitioners", Copyright Springer-Verlag, Pages 87-121

[46] Jamil, T. "The Rijndael algorithm” Potentials, IEEE (Volume:23, Issue: 2) pp. 36 - 38, 2004

[47] Hanem M. El-Sheikh; Omayma A. El-Mohsen; TalaatElgarf; AbdelhalimZekry, "A New Approach for Designing KeyDependent S-Box Defined over GF (2^4) in AES “International Journal of Computer Theory and Engineering Vol. 4, No. 2, April 2012.

[48] Iqtadar Hussain; Tariq Shah; Hasan Mahmood,” A New Algorithm to Construct Secure Keys for AES “Int. J. Contemp. Math. Sciences, Vol. 5, 2010, no. 26, $1263-1270$

[49] Kazys KAZLAUSKAS; GytisVaicekauskas; Robertas SMALIUKAS; “An Algorithm for Key-Dependent S-Box Generation in Block Cipher System "INFORMATICA, 2015, Vol. 26, No. 1, 51-65.

[50] Hongjun Wu; Bart Preneel, "AEGIS: A Fast Authenticated Encryption Algorithm” Springer Berlin Heidelberg, Selected Areas in Cryptography -- SAC 2013, Lecture Notes in Computer Science Volume 8282, 2014, pp. 185-201

[51] Jian Guo; Thomas Peyrin; Axel Poschmann; Matt Robshaw, "The LED block cipher, "Cryptographic Hardware and Embedded Systems - CHES 2011, Lecture Notes in Computer Science Volume 6917, 2011, pp 326-341

[52] Daesung Kwon, Jaesung Kim, Sangwoo Park, Soo Hak Sung, YaekwonSohn, Jung Hwan Song, YongjinYeom, E-Joong Yoon, Sangjin Lee, Jaewon Lee, Seongtaek Chee, Daewan Han, Jin Hong, "New Block Cipher: ARIA," Springer Berlin Heidelberg, Information Security and Cryptology - ICISC 2003, Lecture Notes in Computer Science Volume 2971, 2004, pp 432-445

[53] Joan Daemen, Lars Knudsen and Vincent Rijmen, "The block cipher Block" Springer Berlin Heidelberg, Fast Software Encryption Lecture Notes in Computer Science Volume 1267, 1997, pp.149-165

[54] Takeshi Shimoyama, Hitoshi Yanami, Kazuhiro Yokoyama, Masahiko Takenaka, Kouichi Itoh, Jun Yajima, Naoya Torii, Hidema Tanaka, "The Block Cipher SC2000", Springer Berlin Heidelberg, Fast Software Encryption, Lecture Notes in Computer Science Volume 2355, 2002, pp. 312-327

[55] Eli Biham, Ross Anderson, Lars Knudsen, "Serpent: A New Block Cipher Proposal”, Springer Berlin Heidelberg, Fast Software Encryption Volume 1372 of the series Lecture Notes in Computer Science pp 222-238. 1998

[56] Bruce Schneier, John Kelsey, “Unbalanced Feistel networks and block cipher design”, Springer Berlin Heidelberg, Fast Software Encryption, Lecture Notes in Computer Science Volume 1039, pp. 121-144, 1996

[57] TaizoShirai, KyojiShibutani, Toru Akishita, Shiho Moriai, Tetsu Iwata, "The 128-Bit Blockcipher CLEFIA” Springer Berlin Heidelberg, Fast Software Encryption, Lecture Notes in Computer Science Volume 4593, 2007, pp. 181-195

[58] Deukjo Hong, Jaechul Sung, Seokhie Hong, Jongin Lim,Sangjin Lee, Bon-Seok Koo,Changhoon Lee, Donghoon Chang, Jesang Lee, KitaeJeong, Hyun Kim, Jongsung Kim,Seongtaek Chee. "HIGHT: A New Block Cipher Suitable for LowResource Device”,Springer Berlin Heidelberg, Cryptographic Hardware and Embedded Systems - CHES 2006, Volume 4249 of the series Lecture Notes in Computer Science pp 46-59

[59] Matt Blaze, Bruce Schneier, "The MacGuffin block cipher algorithm”, Springer Berlin Heidelberg, Fast Software Encryption, Lecture Notes in Computer Science Volume 1008, pp. 97-110,1995

[60] Poonam Jindal, Brahmjit Singh, “A Survey on RC4 Stream Cipher, I. J. Computer Network and Information Security, 2015, 7, 37-45, http://www.mecs-press.org/

\section{AUTHORS PROFILE}

DAYANANDA LAL N , Research Scholar, Department of Electronics and Communication Engineering, Dr. MGR Educational and Research Institute University, Chennai, India Email id: dayanandlal@gmail.com

Dr. SENTHIL KUMAR.K, Professor, Department of Electronics and Communication Engineering, Dr. MGR Educational and Research Institute University, Chennai, India Email id: ksenthilkumar@drmgrdu.ac.in 